\title{
Hospitalización en pacientes centenarios en Galicia en el periodo 2004-2013
}

\author{
Hospitalization in centenarian patients in Galicia in the period 2004-2013
}

\author{
Eva Romay-Lema, Juan Carlos Piñeiro-Fernández, Pablo Ventura-Valcárcel, Iria Iñiguez-Vázquez, \\ Raquel Gómez-Méndez, Ramón Rabuñal-Rey, Rafael Monte-Secades \\ Hospital Universitario Lucus Augusti
}

\section{Resumen}

Objetivo: Describir la tendencia temporal y el perfil clínico de los centenarios que ingresan en los hospitales gallegos.

Método: Análisis del Registro de Altas del Sistema Nacional de Salud en el periodo 2004 - 2013.

Resultados: Se registraron 1.373 ingresos, con una edad media de $101,2 \pm 1,4$. El $73,7 \%$ fueron mujeres. El número de ingresos por año se incrementó con el tiempo (0,31\% de los ingresos en 2004 versus $0,69 \%$ en 2013). Ourense y Lugo fueron las provincias donde hubo más ingresos en proporción con la población atendida. Más de la mitad de los pacientes fueron atendidos por los Servicios de Medicina Interna. Los centenarios ingresaron a través de Servicio de Urgencias, y el motivo de ingreso fue médico en el 84,7\%, constituyendo el GRD (Grupos relacionados por el diagnóstico) más frecuente las infecciones respiratorias. En los varones hubo una mayor incidencia de enfermedades respiratorias, mientras que las mujeres presentaron mayor incidencia de fracturas de cadera. La estancia media fue de 8,6 7 días, alcanzando una mortalidad de casi el $30 \%$ y la tasa de reingresos al año fue del $7,8 \%$.

Conclusiones: Se observa un incremento progresivo del número de ingresos de pacientes centenarios en los últimos años, fundamentalmente por patología médica. Presentan una mortalidad elevada y reingresan con frecuencia.

Palabras clave: centenarios, epidemiologia, ingreso hospitalario.

\section{Introducción}

Los avances en el ámbito sociosanitario, el aumento de la esperanza de vida y la disminución de la natalidad trajeron consigo, entre otros factores, el envejecimiento progresivo de las sociedades desarrolladas en las últimas décadas. Un rasgo característico del envejecimiento demográfico moderno es el aumento sin precedentes en la proporción de personas centenarias. De mantenerse las tendencias actuales, la población centenaria española pasaría de las 13.551 personas en el año 2014, a más de 372.000 en el año 20641. Este fenómeno se acentúa en Galicia, una de las comunidades autónomas españolas con mayor índice de senectud. Ya se han publicado datos parciales que indican que existe un incremento de la demanda sanitaria por parte de este colectivo en los últimos años ${ }^{2}$, pero sin embargo este grupo de población sigue poco estudiado.

El objetivo del presente trabajo es analizar los ingresos en los hospitales gallegos de pacientes centenarios, para conocer su perfil clínico y comprobar si se confirman las tendencias anteriormente mencionadas.

\section{Abstract}

Objective: To describe the clinical profile of centenarians admitted to galician hospitals.

Method: Review of Discharge Register of the National Health System in the period 2004-2013.

Results: The sampled included 1,373 admissions, with mean age of $101.2 \pm 1.4$.

$73.7 \%$ were women. The number of admissions per year increased over time (0.31 \% in 2004 versus $0.69 \%$ in 2013). Ourense and Lugo were the provinces with more admissions in proportion to the reference population. Internal Medicine Services attended more than half of patients. Centenarians were admitted through the emergency department, and the reason for admission was medical in $84.7 \%$, being the most frequent DRG (Diagnosis-Related Group) respiratory infections. There was a higher incidence of respiratory diseases in males, while women had a higher incidence of hip fractures. The mean length of stay was $8.6 \pm 7$ days, with a mortality of almost $30 \%$. The readmission rate was $7.8 \%$.

Conclusions: There is a progressive increase in the number of centenarians admissions in the lasts years, mainly due to medical conditions. They have a high mortality and are frequently readmitted.

Key words: centenarian, epidemiology, hospital admission.

\section{Método}

A través del Registro de Altas CMBD de Hospitalización y Atención Ambulatoria Especializada del Ministerio de Sanidad, Servicios Sociales e Igualdad ${ }^{3}$ se obtuvieron los datos correspondientes a las altas hospitalarias de todos los pacientes mayores de 100 años atendidos en los hospitales públicos gallegos pertenecientes al Servicio Nacional de Salud entre 2004 y 2013 (ambos incluidos).

Se recogieron de cada episodio de ingreso los siguientes datos clínicos y demográficos: el sexo, la edad, la provincia de procedencia, el servicio de ingreso, el carácter del mismo (urgente o programado), la tasa de reingresos y el tipo de alta. Se identificó para cada paciente un GRD (Grupo Relacionado por el Diagnóstico), su correspondiente Categoría Diagnóstica Mayor (agrupaciones excluyentes de los GRD clasificadas por sistemas orgánicos), y el coste estimado para cada GRD.

Además, en la página web del Instituto Gallego de Estadística ${ }^{4}$ se obtuvo el número total de ingresos de la población gallega en los últimos 10 años y se comparó con el número de pacientes centenarios ingresados. 
Figura 1. Distribución por sexo y provincia de los centenarios ingresados en el periodo 2004-2013

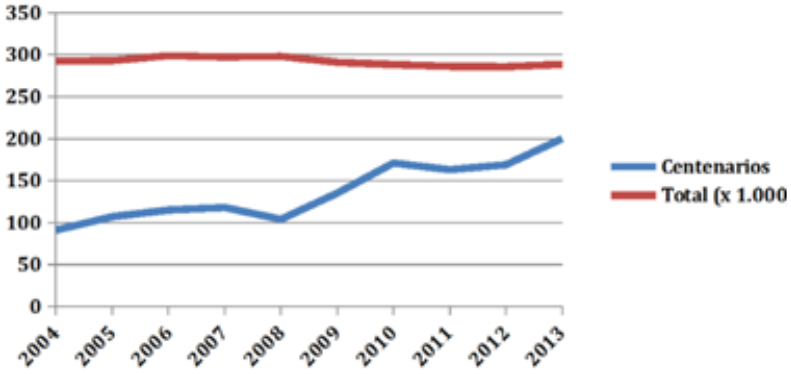

Se realizó un estudio descriptivo de las variables incluidas en el estudio. Las variables cualitativas fueron expresadas como valor absoluto y porcentaje. Las variables cuantitativas se expresaron como media y su correspondiente desviación estándar. En el análisis estadístico se utilizó la técnica Chi cuadrado con la corrección de Yates cuando fue preciso, en la comparación de las variables cualitativas. En la comparación de dos variables cuantitativas se utilizó el test T de Student, previa comprobación de normalidad. El nivel de significación estadística fue $p<0,05$. En el análisis se utilizó el paquete estadístico SPSS 18.

\section{Resultados}

Se registraron 1.373 ingresos de centenarios, con una edad media de 101,2 $\pm 1,4$ años, de los cuales un 73.7\% fueron mujeres (figura 1). Su distribución por año de ingreso y provincia se muestran en la figura 2. El número de centenarios atendidos por año se incrementó de forma progresiva en el tiempo, duplicándose a lo largo de los diez años estudiados (0,31\% de los ingresos en 2004 versus $0,69 \%$ en 2013). Este aumento fue independiente del número de ingresos de
Figura 2. Evolución del número de ingresos de pacientes centenarios respecto a los ingresos totales en el periodo 2004-2013

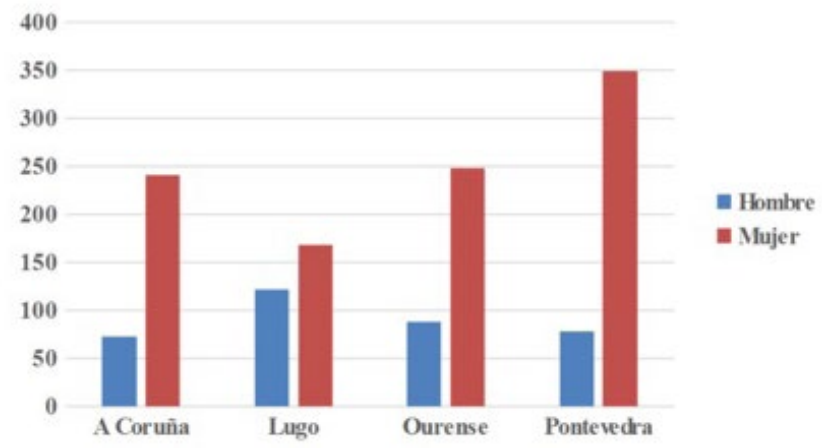

la población total gallega, que incluso disminuyó en un 1,5\% en la década analizada (tabla 1).

El motivo de ingreso en un $84.7 \%$ de los casos fue de causa médica. En la tabla 2 se muestran las Categorías de Diagnostico Mayor. Las más frecuentes fueron las patologías de Ios sistemas respiratorio y circulatorio. No hubo diferencias significativas entre sexos en la frecuencia de las distintas categorías salvo en el caso de las Enfermedades y trastornos del sistema respiratorio (hombres $40,7 \%$ vs mujeres $32,7 \%, p=0.006$ ) y las Enfermedades y trastornos del sistema musculoesquelético (hombres $7,5 \%$ vs mujeres $13,2 \%$, $\mathrm{p}=0.003$ ).

Los 10 GRDs más frecuentes en la población centenaria se exponen en la tabla 3. Las infecciones respiratorias causan el 20,9\% de los ingresos, seguidos en frecuencia por la insuficiencia cardíaca (13\%). Se realizaron 54 procedimientos sobre cadera y fémur, constituyendo este el único GRD quirúrgico dentro de los 10 más frecuentes.

La vía más común de acceso al hospital fue a través del Servicio de Urgencias (97,8\%), tanto si habían acudido por enfermedad médica como por problemas quirúrgicos. Los

Tabla 1. Distribución de los ingresos por provincia y año, y tasa calculada (por 1.000 ingresos)

\begin{tabular}{|c|c|c|c|c|c|c|c|c|c|c|c|c|}
\hline & \multicolumn{3}{|c|}{ A Coruña } & \multicolumn{3}{|c|}{ Lugo } & \multicolumn{3}{|c|}{ Ourense } & \multicolumn{3}{|c|}{ Pontevedra } \\
\hline Año & $\begin{array}{l}\mathrm{N}^{0} \text { total de } \\
\text { ingresos }\end{array}$ & $\begin{array}{l}\mathrm{N}^{0} \text { de ingresos } \\
\text { en centenarios }\end{array}$ & Tasa & $\begin{array}{l}\mathrm{N}^{0} \text { total de } \\
\text { ingresos }\end{array}$ & $\begin{array}{l}N^{0} \text { de ingresos } \\
\text { en centenarios }\end{array}$ & Tasa & $\begin{array}{l}\mathrm{N}^{0} \text { total de } \\
\text { ingresos }\end{array}$ & $\begin{array}{l}\mathrm{N}^{0} \text { de ingresos } \\
\text { en centenarios }\end{array}$ & Tasa & $\begin{array}{l}\mathrm{N}^{0} \text { total de } \\
\text { ingresos }\end{array}$ & $\begin{array}{c}\mathrm{N}^{0} \text { de } \\
\text { ingresos en } \\
\text { centenarios }\end{array}$ & Tasa \\
\hline 2004 & 129.808 & 30 & 0,23 & 37.000 & 22 & 0,59 & 36.598 & 21 & 0,57 & 91.415 & 18 & 0,20 \\
\hline 2005 & 129.641 & 13 & 0,10 & 37.185 & 22 & 0,59 & 36.292 & 38 & 1,05 & 92.177 & 32 & 0,35 \\
\hline 2006 & 130.652 & 34 & 0,26 & 38.313 & 25 & 0,65 & 37.658 & 28 & 0,74 & 94.545 & 28 & 0,30 \\
\hline 2007 & 129.203 & 25 & 0,19 & 38.371 & 12 & 0,31 & 37.103 & 39 & 1,05 & 94.953 & 41 & 0,43 \\
\hline 2008 & 128.373 & 16 & 0,12 & 39.022 & 22 & 0,56 & 35.967 & 26 & 0,72 & 96.880 & 40 & 0,41 \\
\hline 2009 & 126.350 & 35 & 0,28 & 37.666 & 29 & 0,77 & 35.013 & 31 & 0,89 & 94.162 & 40 & 0,42 \\
\hline 2010 & 125.006 & 37 & 0,30 & 36.065 & 46 & 1,28 & 35.267 & 37 & 1,05 & 94.144 & 49 & 0,52 \\
\hline 2011 & 125.604 & 35 & 0,28 & 34.966 & 39 & 1,12 & 34.924 & 35 & 1,00 & 92.678 & 54 & 0,58 \\
\hline 2012 & 125.801 & 41 & 0,33 & 34.775 & 31 & 0,89 & 34.776 & 41 & 1,18 & 92.301 & 56 & 0,61 \\
\hline 2013 & 125.008 & 48 & 0,38 & 34.571 & 42 & 1,21 & 37.250 & 40 & 1,07 & 93.631 & 69 & 0,74 \\
\hline Promedio & & & 0,25 & & & 0,80 & & & 0,93 & & & 0,46 \\
\hline
\end{tabular}

Cifras de población obtenidas del Instituto Galego de Estatística (www.ige.eu) 
Tabla 2. Distribución por sexos de los diagnósticos realizados al alta hospitalaria en pacientes centenarios

\begin{tabular}{|c|c|c|c|}
\hline Categoría de diagnóstico mayor & $\begin{array}{l}\text { Varones } \\
(\mathrm{n}=361)\end{array}$ & $\begin{array}{l}\text { Mujeres } \\
(n=1012)\end{array}$ & $\mathrm{p}^{*}$ \\
\hline Enfermedades y trastornos del sistema respiratorio & $147(40.7 \%)$ & $331(32.7 \%)$ & 0.006 \\
\hline Enfermedades y trastornos del sistema circulatorio & $58(15.5 \%)$ & $180(17,8 \%)$ & 0.32 \\
\hline Enfermedades y trastornos del sistema musculoesquelético y conectivo & $27(7,5 \%)$ & $134(13,2 \%)$ & 0.003 \\
\hline Enfermedades y trastornos del sistema nervioso & $27(7,5 \%)$ & $85(8,4 \%)$ & 0.58 \\
\hline Enfermedades y trastornos del sistema digestivo & $28(7,8 \%)$ & $77(7,6 \%)$ & 0.92 \\
\hline Enfermedades y trastornos del riñón y tracto urinario. & $28(7,8 \%)$ & $54(5,3 \%)$ & 0.09 \\
\hline Enfermedades y trastornos del sistema hepatobiliar y páncreas & $11(3 \%)$ & $50(4.9 \%)$ & 0.17 \\
\hline Trastornos endocrinos, de la nutrición y del metabolismo & $5(1,4 \%)$ & $22(2.2 \%)$ & 0.48 \\
\hline Otros & $30(8,3 \%)$ & $79(7,8 \%)$ & 0.76 \\
\hline
\end{tabular}

${ }^{*}$ Chi cuadrado. Datos expresados en $n^{\circ}$ y porcentaje (\%)

pacientes fueron atendidos de forma predominante en los Servicios de Medicina Interna (54,4\%), seguidos por los Servicios de Geriatría y de Traumatología, que asumieron un $13,8 \%$ y $10,3 \%$ de los ingresos respectivamente. (Tabla 4) La mayoría de los enfermos tuvieron una estancia hospitalaria prolongada con una media de $8,6 \pm 7$ días. Fallecieron durante el ingreso 402 pacientes $(29,3 \%)$, sin diferencias estadísticamente significativas entre sexos. La tasa de reingresos (en el mismo centro y año, en los 30 días siguientes al alta) fue del 7,8\%.

El Coste calculado por episodio de hospitalización fue de $4.653 \pm 2.377$ euros, siendo superior en las mujeres (4.782 \pm 2.525 vs $4.294 \pm 1.858, p<0.001)$

\section{Discusión}

Los datos epidemiológicos indican un progresivo aumento de la población centenaria, lo cual implica que cada vez será más frecuente atender en el hospital a pacientes extremadamente ancianos. Es evidente que la atención a estos pacientes no puede ser igual a la prestada a población más joven, tanto por su corta expectativa de vida como por la potencial agresividad de las intervenciones. Por tanto será importante conocer el perfil de estos pacientes para poder planificar adecuadamente su atención, adecuándola a su situación de forma que se le administre el tratamiento más correcto sin caer ni el encarnizamiento terapéutico ni en el nihilismo.

En el presente estudio confirmamos un incremento progresivo del número de ingresos de pacientes centenarios en los últimos años. Al igual que en la mayoría de estudios realizados en centenarios se observa una mayor proporción de mujeres ${ }^{4}$. Aunque en números absolutos la provincia con mayor incidencia de ingresos de centenarios es Pontevedra, si calculamos la tasa de ingresos por población atendida, ocupan los primeros puestos Ourense y Lugo, dato que concuerda con las pirámides poblacionales correspondientes, que demuestran que alrededor del $30 \%$ de la población de Galicia oriental se sitúa por encima de los 65 años ${ }^{5}$.
Tabla 3. GRDs más frecuentes en la población centenaria

\begin{tabular}{|l|c|}
\hline GRD & $\%$ \\
\hline $\begin{array}{l}\text { Neumonía simple y otros trastornos } \\
\text { respiratorios excepto bronquitis y asma con } \\
\text { complicación mayor }\end{array}$ & $199(14,5 \%)$ \\
\hline $\begin{array}{l}\text { ICC y arritmia cardíaca con complicación } \\
\text { mayor }\end{array}$ & $77(5,6 \%)$ \\
\hline Insuficiencia cardíaca y shock & $69(5 \%)$ \\
\hline $\begin{array}{l}\text { Otros diagnósticos del aparato respiratorio con } \\
\text { complicación }\end{array}$ & $60(4,4 \%)$ \\
\hline $\begin{array}{l}\text { Procedimientos sobre cadera y fémur excepto } \\
\text { articulación mayor edad> } 17 \text { sin complicación } \\
\text { 0 comorbilidad }\end{array}$ & $54(3,9 \%)$ \\
\hline $\begin{array}{l}\text { Infecciones respiratorias excepto neumonía } \\
\text { con complicación mayor }\end{array}$ & $49(3,6 \%)$ \\
\hline $\begin{array}{l}\text { Otros trastornos del Sistema nervioso excepto } \\
\text { AlT, convulsiones y cefalea }\end{array}$ & $39(2,8 \%)$ \\
\hline $\begin{array}{l}\text { Neumonía simple y pleuritis edad >17 con } \\
\text { complicación 0 comorbilidad }\end{array}$ & $38(2,8 \%)$ \\
\hline Ictus con infarto & $35(2,5 \%)$ \\
\hline Edema pulmonar e insuficiencia respiratoria & $33(2,4 \%)$ \\
\hline
\end{tabular}

Tabla 4. Distribución por servicios de los pacientes centenarios ingresados

\begin{tabular}{|l|c|}
\hline Servicio & Ingresos \\
\hline Medicina Interna & $747(54,4 \%)$ \\
\hline Geriatría & $190(13,8 \%)$ \\
\hline Traumatología & $142(10,3 \%)$ \\
\hline Cirugía general & $55(4 \%)$ \\
\hline Digestivo & $38(2,8 \%)$ \\
\hline Cardiología & $18(1,3 \%)$ \\
\hline Neumología & $15(1,1 \%)$ \\
\hline Urología & $14(1 \%)$ \\
\hline Neurología & $13(0,9 \%)$ \\
\hline Cirugía vascular & $9(0,7 \%)$ \\
\hline Otros & $132(9,7 \%)$ \\
\hline
\end{tabular}

Datos expresados en $n^{0}$ y porcentaje (\%) 
La mayoría de los ingresos son de causa médica. Los diagnósticos al alta hospitalaria son similares a los descritos en otras series de centenarios analizadas en España, siendo las afecciones más frecuentes las infecciones respiratorias, los eventos cardiovasculares y las fracturas. En un estudio realizado en pacientes nonagenarios ingresados en los Servicios de Medicina Interna a nivel nacional, 8 de los 10 GRDs más frecuentes coinciden con los de nuestro estudio ${ }^{6}$. Estos diagnósticos traducen la prevalencia de patología cardiovascular de esta población, y su cronicidad justifica la elevada proporción de reingresos, al igual que la elevada mortalidad. Respecto al tipo de alta, se observa una mortalidad elevada (casi un 30\%), sin presentar diferencias significativas entre sexos. Estos resultados difieren de los descritos en otras series, donde el sexo masculino se asoció de forma independiente a una mayor mortalidad? .

En cuanto a la distribución por servicios, cabe destacar que más de la mitad de los pacientes son atendidos por los servicios de Medicina Interna, algo que parece lógico dado que se trata de pacientes que por su elevada comorbilidad precisan un enfoque global y multidisciplinar como el que ofrece el internista.

Los ingresos en los servicios quirúrgicos son poco frecuentes, siendo el más representativo la fractura de cadera, que consideramos que es lógico y está influida por la fragilidad de estos pacientes y la osteoporosis secundaria a la avanzada edad y menor actividad física. Como ocurre en otras series descritas previamente, esta patología se describe más frecuentemente en el sexo femenino ${ }^{8}$.

La principal limitación de nuestro estudio es que se apoya en datos extraídos de una base administrativa, lo cual podría comprometer la calidad de los datos, debido que puede ha- ber disparidad entre servicios y centros tanto en la confección como en la interpretación de los informes de alta. Sin embargo, la concordancia de esta base administrativa con un registro clínico ya ha sido demostrada para otras patologías ${ }^{9}$.En conclusión, está aumentando progresivamente la atención hospitalaria a población centenaria, atención por otra parte que se inicia frecuentemente a través de Urgencias, en pacientes con pluripatología y elevada morbimortalidad. Sería aconsejable establecer vías clínicas de atención a estos pacientes para adecuarla a su situación evitando medidas diagnostico terapéuticas inadecuadas para ellos.

\section{Bibliografía}

1. Proyección de la Población de España 2014-2064. http://www.ine.es/inebaseDYN/ propob30278/docs/meto_propob.pdf.

2. Rabuñal Rey R, Monte Secades R, Rigueiro Veloso MT, Casariego Vales EJ, Ibáñez Alonso MD, García Pais MJ. Pacientes centenarios atendidos en un hospital general. Rev Clin Esp. 2002 Jun;202(6):326-8

3. Ministerio de Sanidad, Servicios Sociales e Igualdad. http://www.msssi.gob.es/estadEstudios/estadisticas/estadisticas/estMinisterio/SolicitudCMBD.htm

4. Rabuñal Rey R, Monte Secades R, Veiga Cando MD, Rigueiro Veloso MT, López Díaz MJ, Casariego Vales EJ, Guerrero Lombardía J. Estado de salud de los muy ancianos: situación clínica y funcional de la población centenaria. An Med Interna. 2004 Nov;21(11):543-7

5. Instituto Galego de Estadística. http://www.ige.eu

6. Lázaro M, Marco J, Barba R, Ribera JM, Plaza S, Zapatero A. Nonagenarios en los servicios de Medicina Interna españoles. Rev Esp Geriatr Gerontol. 2012 SepOct;47(5):193-7.

7. Martín-Sánchez FJ, Fernández-Alonso C, Hormigo Al, Jiménez-Díaz G, Roiz H, Bermejo-Boixareu C, Rodríguez-Salazar J, Fernández Pérez C, Gil-Gregorio P. Perfil clínico y mortalidad a 90 días de los pacientes centenarios atendidos en servicios de urgencias hospitalarios. Rev Esp Geriatr Gerontol. 2016 Feb 22. pii: S0211139X(16)00010-X.

8. Rochon PA, Gruneir A, Wu W, Gill SS, Bronskill SE, Seitz DP, Bell CM, Fischer HD, Stephenson AL, Wang X, Gershon AS, Anderson GM. Demographic characteristics and healthcare use of centenarians: a population-based cohort study. J Am Geriatr Soc. 2014 Jan;62(1):86-93.

9. Guijarro R, Montes J, Sanromán C, Monreal M; RIETE Investigators. Venous thromboembolism in Spain. Comparison between an administrative database and the RIETE registry. Eur J Intern Med. 2008 0ct;19(6):443-6. 